\title{
Parameter-Invariant Hierarchical Exclusive Alphabet Design for 2-WRC with HDF Strategy
}

\author{
T. Uřičár̆
}

\begin{abstract}
Hierarchical eXclusive Code (HXC) for the Hierarchical Decode and Forward (HDF) strategy in the Wireless 2-Way Relay Channel (2-WRC) has the achievable rate region extended beyond the classical MAC region. Although direct HXC design is in general highly complex, a layered approach to HXC design is a feasible solution. While the outer layer code of the layered HXC can be any state-of-the-art capacity approaching code, the inner layer must be designed in such a way that the exclusive property of hierarchical symbols (received at the relay) will be provided. The simplest case of the inner HXC layer is a simple signal space channel symbol memoryless mapper called Hierarchical eXclusive Alphabet (HXA). The proper design of HXA is important, especially in the case of parametric channels, where channel parametrization (e.g. phase rotation) can violate the exclusive property of hierarchical symbols (as seen by the relay), resulting in significant capacity degradation. In this paper we introduce an example of a geometrical approach to Parameter-Invariant HXA design, and we show that the corresponding hierarchical MAC capacity region extends beyond the classical MAC region, irrespective of the channel parametrization.
\end{abstract}

Keywords: physical layer network coding, wireless 2-way relay channel, HDF strategy, HXA design.

\section{Introduction}

\subsection{Background and related work}

Communication scenarios based on principles similar to Network Coding (NC) [1] are expected to have great potential for wireless communication networks. Although pure NC operates with a discrete (typically binary) alphabet over lossless discrete channels, its principles can be extended into the wireless domain. Such an extension is however non-trivial, because signal space link models (e.g. the MAC phase in relay communications) lack the simple finite field properties found and used in pure discrete NC. NC-based approaches in the signal space domain are called Physical Network Coding (PNC) or Network Coded Modulation (NCM).

The major benefit of NCM is the possibility to increase the throughput in the MAC phase of bidirectional communication, which is believed to be the bottleneck in the overall system. The strategy where the relay decodes only hierarchical symbols (codewords), which jointly represent information received from both sources, is called the Hierarchical Decode and Forward (HDF) strategy [2,3]. The increased MAC phase throughput of the HDF strategy provides a performance improvement over standard techniques based on the Amplify \& Forward or Joint Decode \& Forward paradigms. The authors of [4] present the simplest realization of HDF strategy with minimal cardinality mapping, which they call "modulo decoding". More general relay output mapping, which also takes into account the possibility of extended cardinality, is introduced in [2].

Only limited code design and capacity region results are available even for the simplest possible scenario of the parametric 2-Way Relay Channel (2WRC), see $[5,6,7,8]$. Lattice-based code construction $[4,9]$ using the principles from [10] is limited to non-parametric Gaussian channels. References [2, 3] present a layered approach to Hierarchical eXclusive Code (HXC) design for parametric 2-WRC, based on the Hierarchical eXclusive Alphabet (HXA). Hierarchical MAC capacity regions for various alphabets, constellation point indexing and channel parametrization are evaluated in [3]. Significant capacity degradation is caused by channel parametrization, which highlights the importance of HXA design resistant to channel parametrization.

\subsection{Goals and contribution of this paper}

The proper design of HXA is critical for overall system performance, especially in the case of parametric 2WRC, where the channel parametrization (e.g. phase rotation) can cause significant capacity degradation. The parametrization should be taken into account in the design process, and HXA resistant to the effects of channel parametrization should be found. One way to achieve this is by designing the HXA with parameter invariant Hierarchical Decision Maps (HDM) at the relay. The first design approaches of this kind (E-PHXC design criteria $[11,12]$ ) have led so far only 
to orthogonal or non-zero mean HXA with unsatisfactory performance (capacity limited by classical MAC).

In this paper, we introduce an example of a geometrical approach to the design of a multi-dimensional HXA which extends the hierarchical MAC capacity region beyond the classical MAC region irrespective of the channel parametrization.

\section{System model and definitions}

We adopt the system model presented in [3]. We consider a parametric wireless 2-WRC system (Fig. 1), which has 3 physically separated nodes (sources A, $\mathrm{B}$ and relay $\mathrm{R}$ ) supporting two-way communication through a common shared relay $\mathrm{R}$. The source for data $\mathrm{A}$ is co-located with the destination for data B, and vice-versa. The transmitted data of each source serves at the same time as Side Information (SI) for the reverse link. The system is wireless, and all transmitted and received symbols are signal space symbols. The channel is assumed to be a linear frequency flat with Additive White Gaussian Noise (AWGN). The whole system operates in a half-duplex mode (one node cannot simultaneously receive and transmit). The overall bi-directional communication is split into a Multiple Access (MAC) phase and a Broadcast (BC) phase.

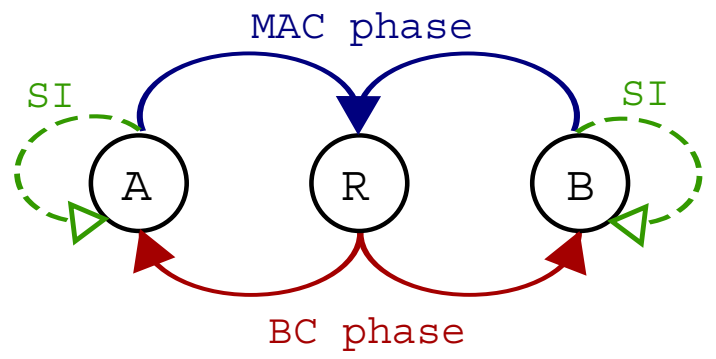

Fig. 1: Model of 2-WRC with side information

\subsection{MAC phase}

Now, we define all formal details. Subscripts $A$ and $B$ denotes variables associated with node $A$ and $B$, respectively. The source data messages are $\mathbf{d}_{A}, \mathbf{d}_{B}$, and they are composed of data symbols $d_{A}, d_{B} \in$ $\mathcal{A}_{d}=\left\{0,1, \ldots, M_{d}-1\right\}$, with alphabet cardinality $\left|\mathcal{A}_{d}\right|=M_{d}$. For notational simplicity, we omit the sequence number indices of the individual symbols. The source node codewords are $\mathbf{c}_{A}, \mathbf{c}_{B}$ with code symbols $c_{A}, c_{B} \in \mathcal{A}_{c},\left|\mathcal{A}_{c}\right|=M_{c}$. The encoding operation is performed by the encoders $\mathcal{C}_{A}, \mathcal{C}_{B}$ with codebooks $\mathbf{c}_{A} \in \mathcal{C}_{A}$ and $\mathbf{c}_{B} \in \mathcal{C}_{B}$. A signal space representation (with an orthonormal basis) of the transmitted channel symbols is $s_{A}=s\left(c_{A}\right), s_{B}=s\left(c_{B}\right)$, $s_{A}, s_{B} \in \mathcal{A}_{s} \subset \mathbb{C}^{N}$. We assume a common channel symbol mapper $\mathcal{A}_{s}(\bullet)$. A signal space representation of the overall coded frame is $\mathbf{s}_{A}\left(\mathbf{c}_{A}\right)$ and $\mathbf{s}_{B}\left(\mathbf{c}_{B}\right)$.
The received useful signal is

$$
u=s_{A}+\alpha s_{B} .
$$

It equivalently represents the parametric channel with both links parametrized according to the flat fading, which is assumed to be constant over the frame. It is obtained by proper common rescaling of the true channel response $u^{\prime}=h_{A} s_{A}+h_{B} s_{B}$ by $1 / h_{A}$ and denoting $\alpha=h_{B} / h_{A}, h_{A}, h_{B}, \alpha \in \mathbb{C}^{1}$. The received signal at the relay is

$$
x=h_{A} u+w
$$

where the circularly symmetric complex Gaussian noise $w$ has variance $\sigma_{w}^{2}$ per complex dimension.

\section{$2.2 \quad$ BC phase}

The relay receives signal $x$ and processes it using a Hierarchical Decode and Forward (HDF) strategy. More details will be given in section 3 . The output codeword and its code symbols are $\mathbf{c}_{R}$ and $c_{R}$. These are mapped into signal space channel symbols $v \in \mathcal{A}_{R}$ and signal space codewords $\mathbf{v}$ with the codebook $\mathbf{v} \in \mathcal{C}_{R}$ and are broadcast to destinations $A$ and $B$. At node $B$ (the destination for data $A$ ), the received signal space symbols are

$$
y_{A}=v+w_{A}
$$

where the complex circularly symmetric AWGN $w_{A}$ has variance $\sigma_{A}^{2}$ per complex dimension. We denote the signal space symbols at node A (a destination for data B) similarly $y_{B}=v+w_{B}$.

\section{Hierarchical exclusive code}

\subsection{Hierarchical decode and forward strategy}

The HDF strategy is based on relay processing, which fully decodes the Hierarchical Data (HD) message $\mathbf{d}_{A B}\left(\mathbf{d}_{A}, \mathbf{d}_{B}\right)$ and sends out the corresponding codeword $\mathbf{v}=\mathbf{v}\left(\mathbf{d}_{A B}\right)$ which represents the original data messages $\mathbf{d}_{A}$ and $\mathbf{d}_{B}$ only through the exclusive law [6]

$$
\begin{aligned}
& \mathbf{v}\left(\mathbf{d}_{A B}\left(\mathbf{d}_{A}, \mathbf{d}_{B}\right)\right) \neq \mathbf{v}\left(\mathbf{d}_{A B}\left(\mathbf{d}_{A}^{\prime}, \mathbf{d}_{B}\right)\right), \forall \mathbf{d}_{A} \neq \mathbf{d}_{A}^{\prime}, \\
& \mathbf{v}\left(\mathbf{d}_{A B}\left(\mathbf{d}_{A}, \mathbf{d}_{B}\right)\right) \neq \mathbf{v}\left(\mathbf{d}_{A B}\left(\mathbf{d}_{A}, \mathbf{d}_{B}^{\prime}\right)\right), \forall \mathbf{d}_{B} \neq \mathbf{d}_{B}^{\prime} .
\end{aligned}
$$

The hierarchical data is a joint representation of the data from both sources such that they uniquely represent one data source given full knowledge of the other source. Assuming that destination node $B$ has perfect Side Information (SI) on the node's own data $\mathbf{d}_{B}$ it can then decode the message $\mathbf{d}_{A}$ (and similarly for node $A$ ). Data $\mathbf{d}_{B}$ will be called complementary data from the perspective of the data $\mathbf{d}_{A}$ operations. The SI on the complementary data will be denoted as Complementary SI (C-SI) [3]. A code (codebook) satisfying the exclusive law at the signal space codeword level (for the HD messages) is called a Hierarchical eXclusive Code (HXC) [3]. We will denote the mapping satisfying the exclusive law by the operator $\mathcal{X}(\bullet, \bullet)$. 
Unlike the standard relaying techniques based on the Joint Decode \& Forward paradigm, where the NC approach is used mainly for a BC phase of communication, in the hierarchical approach (HDF) the hierarchical data is directly obtained from the received signal observations without the need to decode the individual data streams. The fact that only the hierarchical data (not the individual data streams) is decoded at the relay in the MAC phase allows the system throughput to be increased above the constraints given by the classical MAC region. To facilitate this potential throughput benefit, the MAC stage encoding must be such that the observation at the relay allows direct HXC mapping to the hierarchical data. In other words, alongside the complete signal path (MAC and $\mathrm{BC})$ the coding must always be $H X C$ w.r.t. the hierarchical data [3].

Throughout this paper we assume only the HXC with minimal cardinality of the relay hierarchical codebook $\left(\left|\mathcal{C}_{R}\right|=\max \left(\left|\mathcal{C}_{A}\right|,\left|\mathcal{C}_{B}\right|\right)\right)$, which requires that both nodes $(A, B)$ have perfect $\mathrm{C}$-SI on the complementary data. A general discussion on the relay hierarchical codebook cardinality can be found in [3].

\subsection{Layered HXC design for perfect C-SI}

Direct design of the HXC codebook $\mathcal{C}_{R}$ providing the mapping $\mathbf{v}\left(\mathbf{d}_{A B}\left(\mathbf{d}_{A}, \mathbf{d}_{B}\right)\right)$ is evidently highly complex. An alternative approach based on layered $H X C$ design is presented in $[2,3]$. Layered HXC (Fig. 2) consists of the outer layer (error correcting) code and the inner layer (closer to the channel symbols), which provides the exclusive property of the hierarchical symbols. The outer layer code can be an arbitrary state-of-the-art capacity achieving code (e.g. turbo code or LDPC), and the inner layer can be designed (in the simplest case) as a simple signal space channel symbol memoryless mapper. An alphabet memoryless mapper

$$
c_{A B}=\mathcal{X}_{c}\left(c_{A}, c_{B}\right)
$$

fulfilling the exclusive law will be called a Hierarchical eXclusive Alphabet (HXA). The entire model of the layered system can be found e.g. in [3].

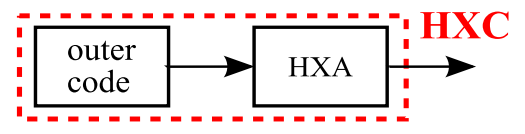

Fig. 2: Layered Hierarchical eXclusive Code

Theorems in [2] show the viability of the layered approach to HXC design. The capacity is alphabet constrained by Hierarchical-MAC (H-MAC) channel hierarchical symbols and is achievable by the outer standard channel code $\mathcal{C}_{A}=\mathcal{C}_{B}=\mathcal{C}$ combined with inner symbol-wise HXA $\mathcal{A}_{s}$. The H-MAC rate region has a rectangular shape

$$
R_{A}=R_{B}=R_{A B} \leq I\left(c_{A B} ; x\right) .
$$

The exclusive property at the symbol level allows simple determination of the soft per-symbol measure decoding metric for hierarchical symbols at the relay. It can either be directly used by the full hierarchical relay decoder or properly source encoded and sent on a per-symbol basis without decoding.

\section{Parameter-invariant HXA}

The complexity of the proper HXA design increases in the case of layered HXC design for a parametric channel. Some specific channel parameter values can cause the signal space points corresponding to different hierarchical symbols to fall into the same useful signal (1) and thereby break the exclusive property, resulting in significant capacity degradation [3]. One possible solution to this inconvenience is to take the channel parametrization into account inherently from the beginning of the HXA design, e.g. by forcing the Hierarchical Decision Maps (HDM) at the relay to be invariant to channel parameter $\alpha$ :

$$
\mathcal{X}_{s(\alpha)}\left(s_{A}, s_{B}\right)=\mathcal{X}_{s}\left(s_{A}, s_{B}\right), \quad \forall \alpha
$$

HXAs that have the HDM invariant to channel parametrization will be called Parameter-Invariant HXAs (PI-HXA).

\subsection{E-PHXC design criteria}

A first attempt to design the PI-HXA for the layered $\mathrm{HXC}$ in 2-WRC was given by the Extended Parametric HXC (E-PHXC) design criteria [11]. These criteria utilize the criterion for the $\alpha$-invariant hierarchical decision region pairwise boundary $\mathcal{R}^{k l}$ (i.e. the decision region boundary between the useful signal pair $u^{k\left(i_{A}, i_{B}\right)}=s_{i_{A}}+\alpha s_{i_{B}}$ and $\left.u^{l\left(i_{A}^{\prime}, i_{B}^{\prime}\right)}=s_{i_{A}^{\prime}}+\alpha s_{i_{B}^{\prime}}\right)$ :

$$
\begin{aligned}
& \left\langle s_{i_{A}}-s_{i_{A}^{\prime}} ; s_{i_{B}}-s_{i_{B}^{\prime}}\right\rangle=0, \\
& \left\langle s_{i_{B}}-s_{i_{B}^{\prime}} ; s_{i_{B}}+s_{i_{B}^{\prime}}\right\rangle=0,
\end{aligned}
$$

to force some subset of the decision region boundaries to be invariant to channel parametrization (see [13] for details). As shown in [12], the E-PHXC design criteria result in HXAs which have all permissible decision region boundaries invariant to channel parametrization, hence the condition for a parameter invariant HDM is naturally satisfied.

Although the E-PHXC design criteria provide a feasible way to design the HXA with parameter invariant HDM, the resulting PI-HXA has a number of drawbacks and only limited performance (see [12] for details). The strictness of the E-PHXC design criteria forces sources $(A, B)$ to use different channel symbol mappers $\left(\mathcal{A}_{A}(\bullet)\right.$ and $\left.\mathcal{A}_{B}(\bullet)\right)$ and the solution leads to mutually orthogonal alphabets or alphabets with nonzero mean and non-equal distance (which is apparently 
not optimal). The solution with mutually orthogonal alphabets has the MAC capacity region equivalent to the classical-MAC decoding [12].

\subsection{Generalized approach to the design}

The strictness of the E-PHXC design criteria (which in turn causes only the orthogonal solution to PI-HXA design to be feasible) is due to the fact that all permissible pairwise boundaries are forced to be parameter invariant. This is obviously not necessary since some decision region boundaries can be "overlaid" by other boundaries or remain somehow "hidden" inside the hierarchical decision region. In such cases, the resulting final shape of the HDM will remain unaffected by these boundaries. Hence these boundaries do not have to be considered by the PI-HXA design criteria. This approach to PI-HXA design should relax the strictness of the design criteria (compared to E-PHXC) and hence non-orthogonal PI-HXAs with the rate region extending the classical MAC region can possibly be found. A comparison of this "generalized" design approach with E-PHXC based design is shown in Fig. 3.

\section{PI-HXA design}

\subsection{Principles of geometrical design}

The derivation of the systematic design criteria (design algorithm) for PI-HXA is still relatively complex. The particular constellation space boundaries of the HDM at the relay result from the selected PI-HXA constellation (i.e. alphabet mapper $\mathcal{A}_{s}$ ), and the design criteria for invariant decision region boundaries directly affect the requirements given on the PI-HXA constellation. This mutual relationship increases the

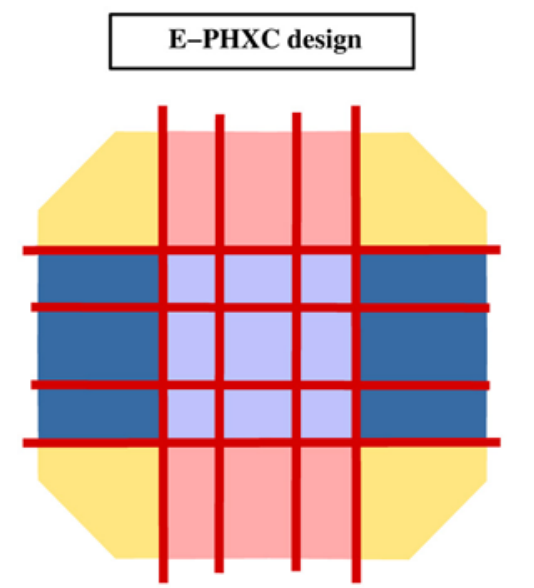

complexity of the systematic solution to PI-HXA design.

We show the viability of the layered HXC solution in parametric channels (i.e. the possibility to find PI-HXA) by introducing some major simplifications which allow a geometric interpretation of the PI-HXA design problem. The idea of the geometric approach to PI-HXA design is based on the "constellation space patterns" of the useful signal $u=s_{A}+\alpha s_{B}$.

Definition 1: A constellation space pattern $\mathcal{U}^{i_{A}}$ is the subspace spanned by the useful signal $u=s_{A}+\alpha s_{B}$ for $s_{A}=s_{i_{A}}, \forall s_{B} \in \mathcal{A}_{s}$ and $\forall \alpha \in \mathbb{C}^{1}$.

The absolute value of the channel parameter $|\alpha| \in$ $(0 ; \infty)$ causes the constellation space patterns to be potentially unbounded. This is the only remaining inconvenience for a simple geometric interpretation of PI-HXA design. As we will show in the following subsection, the constellation space patterns can be effectively bounded by simple processing at the relay.

\subsection{Two-mode relay processing}

The received (useful) signal $u$ is obtained by rescaling the true channel response $\left(u^{\prime}=h_{A} s_{A}+h_{B} s_{B}\right)$ by $1 / h_{A}$. The only purpose of this rescaling is to obtain a simplified expression of the useful signal (1), which is (after rescaling) parametrized only by a single complex channel parameter $\alpha=h_{B} / h_{A}$. It is obvious that the true channel response can alternatively be rescaled by $1 / h_{B}$, hence we can obtain two alternative models of the useful signal $u$ :

$$
\begin{array}{ll}
M_{1}: & u_{M_{1}}=s_{A}+\alpha s_{B} \\
M_{2}: & u_{M_{2}}=\frac{1}{\alpha} s_{A}+s_{B} .
\end{array}
$$

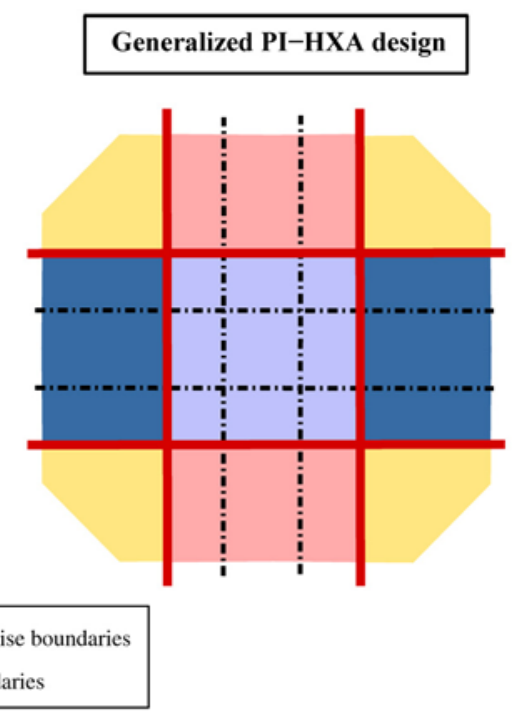

Fig. 3: The final shape of the HDM at the relay is affected only by the pairwise boundaries between different hierarchical codewords (given by a different colour of the region in the figure). The generalized approach to the PI-HXA design requires only these particular boundaries to be invariant to the channel parametrization (unlike E-PHXC) 
This corresponds to two alternative models of the received signal at the relay:

$$
\begin{aligned}
& x_{M_{1}}=h_{A} u_{M_{1}}+w, \\
& x_{M_{2}}=h_{B} u_{M_{2}}+w .
\end{aligned}
$$

The relay can potentially swap these two channel models (respective models of the useful signal) in such a way that the absolute value of the channel parameter ( $\alpha$ for model $M_{1}$ and $\frac{1}{\alpha}$ for $M_{2}$ ) is always less than (or equal to) one. This processing at the relay will be called 2-mode relay processing. If the hierarchical mapping at the relay is "symmetric":

$$
\begin{aligned}
& c_{A B}^{i j}=\mathcal{X}_{c}\left(c_{A}^{i}, c_{B}^{j}\right)=\mathcal{X}_{c}\left(c_{A}^{j}, c_{B}^{i}\right), \\
& \forall i, j \in\left\{1, \ldots\left|\mathcal{A}_{c}\right|\right\}
\end{aligned}
$$

then 2-mode relay processing can be used transparently to both sources A,B (i.e. the sources are not aware which channel model is in use at the relay for the current transmission), and hence it is feasible for the HDF strategy with HXC.

The symmetry of the relay hierarchical mapper allows the relay to swap these two equivalent models of the useful signal (11), (12) transparently to both sources. In this way the relay can ensure that the value of the channel parameter in the useful signal model remains bounded, which in turn affects the subspaces spanned by the useful signals $u_{M_{1}}, u_{M_{2}}$, i.e. the constellation space patterns.

Definition 2: A constellation space pattern $\mathcal{U}^{i_{B}}$ is the subspace spanned by the useful signal $u_{M_{2}}=$ $\frac{1}{\alpha} s_{A}+s_{B}$ for $s_{B}=s_{i_{B}}, \forall s_{A} \in \mathcal{A}_{s}$ and $\forall \alpha \in \mathbb{C}^{1}$.

Definition 3: A bounded constellation space pattern $\mathcal{U}^{\prime i}$ is the subspace given by:

$$
\mathcal{U}^{i}= \begin{cases}\mathcal{U}^{i_{A}} & \text { for }|\alpha| \leq 1 \\ \mathcal{U}^{i_{B}} & \text { for }|\alpha|>1\end{cases}
$$
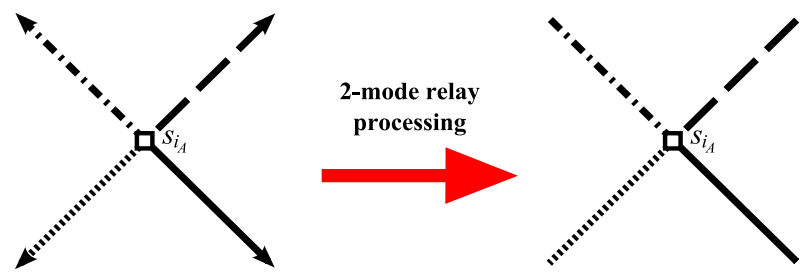

Fig. 4: Example of the constellation space patterns for 2-mode relay processing $\left(\left|\mathcal{A}_{s}\right|=\left|\mathcal{A}_{c}\right|=4\right)$. The particular line style corresponds to the particular $\alpha s_{B}, \forall s_{B} \in \mathcal{A}_{s}$
Table 1: Principles of 2-mode relay processing

\begin{tabular}{cc|ccc}
\hline channel & $|\alpha|$ & $\left|\frac{1}{\alpha}\right|$ & $u$ & $\mathcal{U}^{\prime i}$ \\
\hline \hline$\left|h_{A}\right| \geq\left|h_{B}\right|$ & $\leq 1$ & $\geq 1$ & $u_{M_{1}}$ & $\mathcal{U}^{i_{A}}$ \\
\hline$\left|h_{A}\right|<\left|h_{B}\right|$ & $>1$ & $<1$ & $u_{M_{2}}$ & $\mathcal{U}^{i_{B}}$ \\
\hline
\end{tabular}

Constellation space patterns $\mathcal{U}^{i}$ are effectively bounded (Fig. 4) by simple swapping of the useful signal models at the relay. The only requirement for this 2-mode relay processing is symmetry of the relay hierarchical output mapper (15). We summarize the principles of 2-mode relay processing in Table 1.

\subsection{An example of 2-dimensional PI-HXA}

We assume a real-valued channel symbol memoryless mapper $\mathcal{A}_{s} \subset \mathbb{R}^{2}$ (common to both sources). The channel parameter is complex $\alpha \in \mathbb{C}^{1}$, and the useful signals are $u_{M_{1}}, u_{M_{2}} \in \mathbb{C}^{2}$. The assumption of a real-valued alphabet $\left(\mathcal{A}_{s} \subset \mathbb{R}^{2}\right)$ allows the following simple interpretation of the real and imaginary part of the received useful signal $u_{M_{1}} \in \mathbb{C}^{2}$ (similarly for $\left.u_{M_{2}}\right)$ :

$$
\begin{aligned}
& \Re\left\{u_{M_{1}}\right\}=s_{A}+\Re\{\alpha\} s_{B}, \\
& \Im\left\{u_{M_{1}}\right\}=\Im\{\alpha\} s_{B},
\end{aligned}
$$

which corresponds to the following vector notation:

$$
\begin{aligned}
& \Re\left\{\left[\begin{array}{l}
u_{M_{1}, 1} \\
u_{M_{1}, 2}
\end{array}\right]\right\}=\left[\begin{array}{l}
s_{A, 1} \\
s_{A, 2}
\end{array}\right]+\Re\{\alpha\}\left[\begin{array}{l}
s_{B, 1} \\
s_{B, 2}
\end{array}\right], \\
& \Im\left\{\left[\begin{array}{l}
u_{M_{1}, 1} \\
u_{M_{1}, 2}
\end{array}\right]\right\}=\Im\{\alpha\}\left[\begin{array}{l}
s_{B, 1} \\
s_{B, 2}
\end{array}\right] .
\end{aligned}
$$

It is obvious that the imaginary part of the useful signal $\Im\left\{u_{M_{i}}\right\}$ depends solely on the channel symbols from one source (source B for mode $M_{1}(11)$ and source A for mode $M_{2}(12)$ ). This can be viewed as an additional side information transmission from the corresponding source.

The relay employs 2 -mode processing, hence the corresponding constellation space patterns $\mathcal{U}^{\prime i}$ are bounded. To simplify the design example even more, only the real part of the constellation space patterns $\left(\mathcal{U}_{\operatorname{Re}}^{\prime i}=\Re\left\{\mathcal{U}^{\prime i}\right\}\right)$ will be considered here. These assumptions allow a simple geometric interpretation of the PI-HXA design problem in $\mathbb{R}^{2}$. The common channel symbol mapper $\mathcal{A}_{s}$ cause that for $i_{A}=i_{B}$ the corresponding patterns $\mathcal{U}^{i_{A}}$ and $\mathcal{U}^{i_{B}}$ define identical subspaces. Hence it is sufficient to consider only $\mathcal{U}^{i_{A}}$ for the case $|\alpha| \leq 1$ (it is equivalent to the analysis of $\mathcal{U}^{i_{B}}$ for the case $\left.\left|\frac{1}{\alpha}\right|<1\right)$.

By defining the particular channel symbol mapper $\left(\mathcal{A}_{s}\right)$ we also directly define the corresponding constel- 
lation space patterns $\mathcal{U}^{i_{A}}$. In this case, the geometrical PI-HXA design example turns into a puzzle-like problem of the constellations space pattern $\left(\mathcal{U}_{\mathrm{Re}}^{i_{A}}\right.$ for $\left.i_{A} \in\left\{1,2, \ldots\left|\mathcal{A}_{s}\right|\right\}\right)$ arrangement in $\mathbb{R}^{2}$. The main goal of this "puzzle" is to find a suitable channel symbol mapper $\mathcal{A}_{s}$ and a proper hierarchical eXclusive mapper $c_{A B}^{i j}=\mathcal{X}_{c}\left(c_{A}^{i}, c_{B}^{j}\right)=\mathcal{X}_{s}\left(s_{A}^{i}, s_{B}^{j}\right)$, which will jointly prevent the possibility of violation of the exclusive law for arbitrary channel parameter $\alpha$.

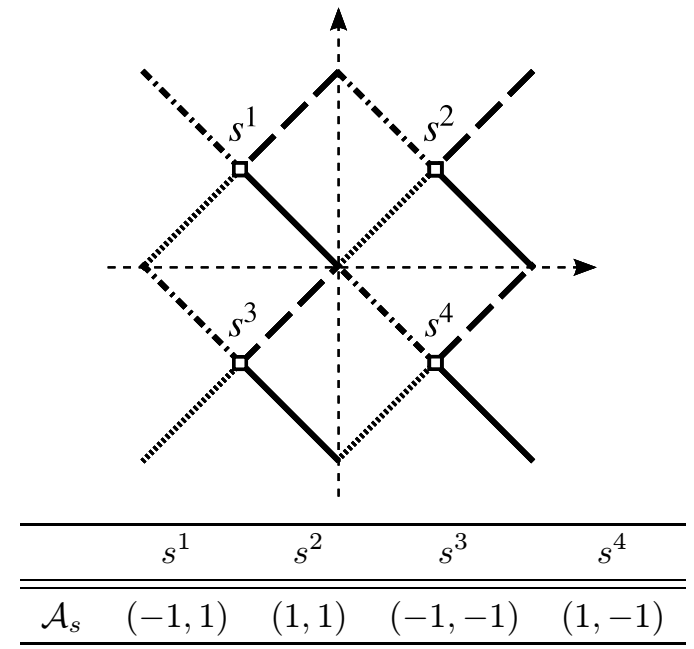

Fig. 5: Channel symbol mapper $\left(\mathcal{A}_{s}\right)$ and the resulting constellation space patterns $\left(\mathcal{U}_{\mathrm{Re}}^{\prime i}\right)$ for the example of PIHXA
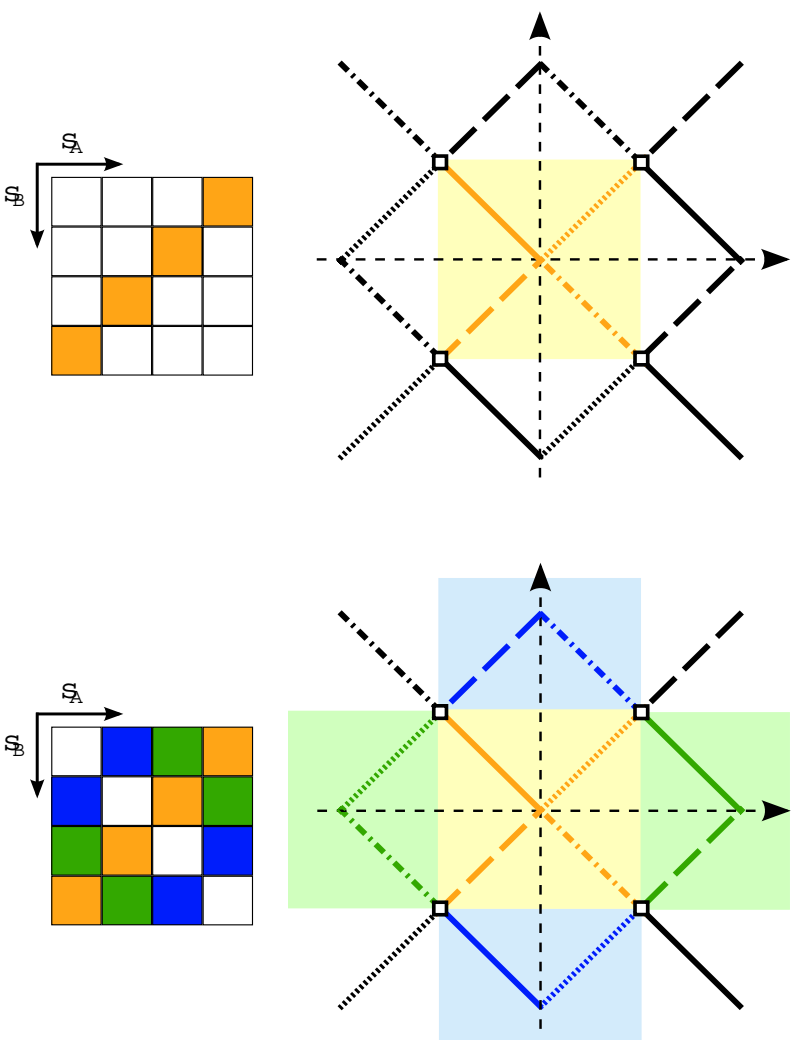

Here we show an example of a two-dimensional 4ary $\left(\left|\mathcal{A}_{s}\right|=\left|\mathcal{A}_{c}\right|=4\right)$ PI-HXA, designed according to the assumptions given in this section. The selected constellation space symbols (i.e. the chosen channel symbol mapper $\mathcal{A}_{s}$ ) and the resulting constellation space patterns $\left(\mathcal{U}_{\mathrm{Re}}^{\prime i}\right)$ are depicted in Fig. 5. The final task of the example of geometrical PI-HXA design is a proper choice of the hierarchical eXclusive mapper. The selection of a suitable hierarchical eXclusive mapper can be visualized as a "colouring" (partitioning) process of the constellation space patterns. A visualization of this colouring process for the example of PI-HXA from Fig. 5 is presented in Fig. 6. The resulting hierarchical eXclusive mapper $\mathcal{X}_{s}\left(s_{A}^{i}, s_{B}^{j}\right)$ corresponds to a bit-wise XOR of the symbol indices $\left(i \in\left\{1,2, \ldots\left|\mathcal{A}_{s}\right|\right\}\right)$.

\section{Numerical results}

To show the viability of the layered HXC design in a parametric 2-WRC with HDF strategy we present some numerical evaluations of the mutual information (capacity) and the minimum squared distance of the example of PI-HXA design from Fig. 5.

\subsection{Mutual information (capacity)}

We evaluate the hierarchical and single-user (alphabet limited cut-set bound) rates (Fig. 7) for an example alphabet $\mathcal{A}_{s}$ (see Fig. 5) and various channel
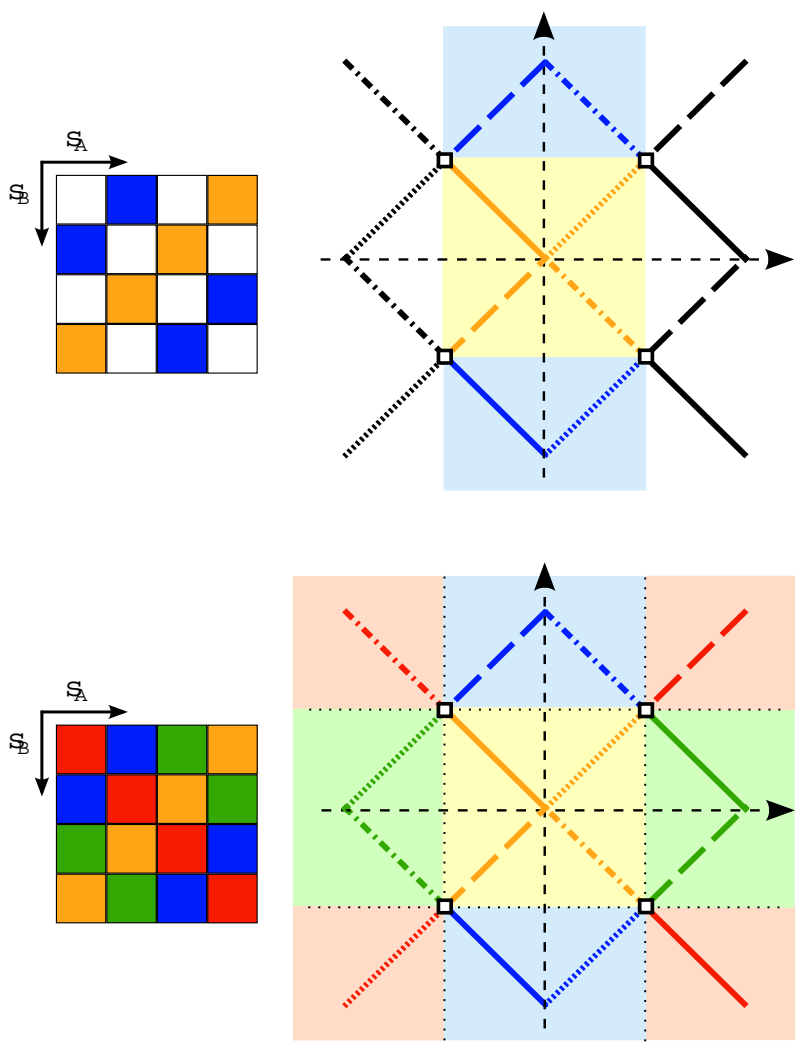

Fig. 6: Design of a suitable hierarchical eXclusive mapper $\mathcal{X}_{s}\left(s_{A}^{i}, s_{B}^{j}\right)$ for the example of PI-HXA from Fig. 5. The resulting hierarchical eXclusive mapper corresponds to a bit-wise XOR of the symbol indices $\left(i \in\left\{1,2, \ldots\left|\mathcal{A}_{s}\right|\right\}\right)$ 


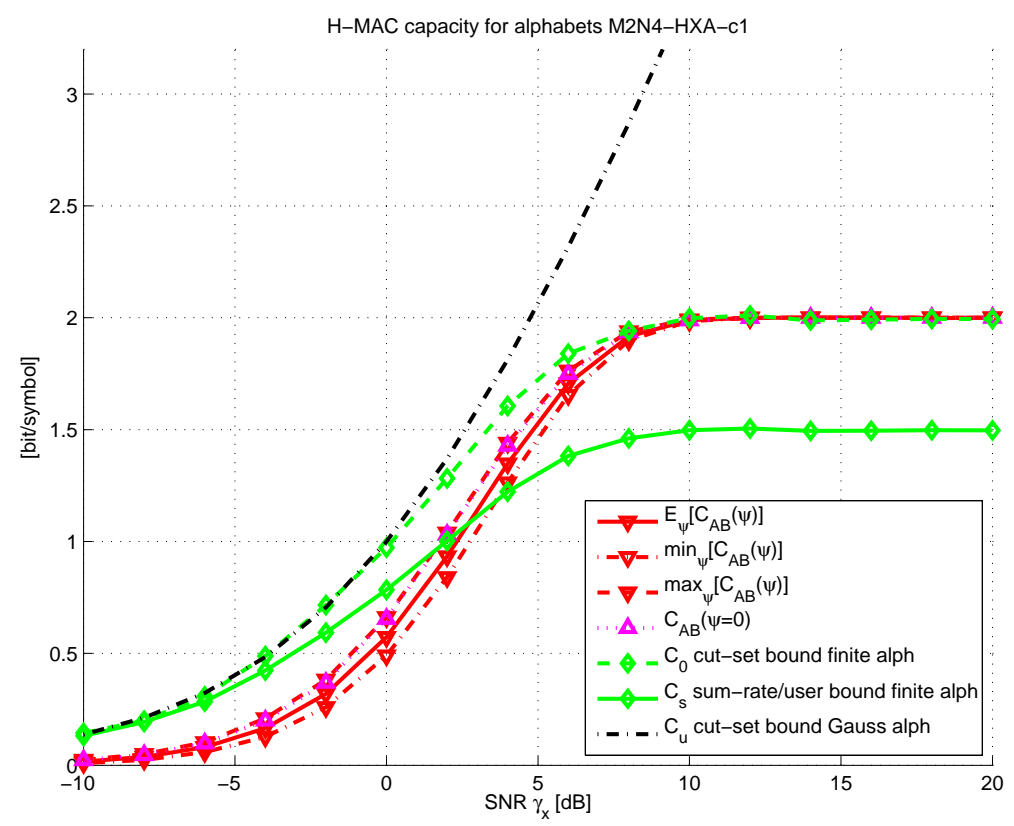

Fig. 7: Capacity (mutual information) for the example of PI-HXA from Fig. 5

parametrization. The Signal-to-Noise Ratio (SNR) is defined as the ratio of the real base-band symbol energy of one source (e.g. A, to have a fair comparison for reference cases) to the noise power spectrum density ratio $\gamma=\left(\overline{\mathcal{E}}_{\mathbf{S}_{A}} / 2\right) / N_{0}$. Assuming orthonormal basis signal space complex envelope representation of the AWGN, we have $\sigma_{w}^{2}=2 N_{0}$ and thus $\gamma=\mathrm{E}\left[\left\|\mathbf{s}_{A}\right\|^{2}\right] / \sigma_{w}^{2}$. The alphabet $\mathcal{A}_{s}$ is indexed by symbols $c_{A}, c_{B} \in\left\{0, \ldots, M_{c}-1\right\}$. The exclusive hierarchical mapping corresponds to a bit-wise XOR of the symbol indices (Fig. 6).

The graph (Fig. 7) shows the classical MAC cutset bounds (1st and 2nd order) related to one user in comparison to the capacity of the HDF strategy with the example of PI-HXA. The HDF capacity is parametrized by the actual relative phase shift of the source-relay channels, while the amplitude is kept constant $|\alpha|$ in our setup (to respect the symmetry of the rates from $\mathrm{A}$ and $\mathrm{B}$ ). We show the minimal, maximal and mean values of the HDF capacity. The results were obtained by the technique shown in [3], where details can be found.

It is obvious from Fig. 7 that the HDF capacity approaches the alphabet constrained cut-set bound limit for medium to high SNR. For SNR values above approximately $2 \mathrm{~dB}$ the capacity outperforms the classical MAC capacity, irrespective of the channel parametrization (relative phase shift of the sourcerelay channel), which has only a minor impact on the resulting performance.

\subsection{Minimum distance}

The minimum distance performance is quite closely connected with the error rate of the whole system [8]. We define the minimum squared distance as:

$$
d_{\min }^{2}=\min _{\mathcal{X}_{s}\left(s_{A}, s_{B}\right) \neq \mathcal{X}_{s}\left(\hat{s}_{A}, \hat{s}_{B}\right)} d_{\left(s_{A}, s_{B}\right)-\left(\hat{s}_{A}, \hat{s}_{B}\right)}^{2},
$$

where $d^{2}$ is the squared Euclidean distance between the useful signal $u=s_{A}+\alpha s_{B}$ and its candidate $\hat{u}=\hat{s}_{A}+\alpha \hat{s}_{B}$ :

$$
d_{\left(s_{A}, s_{B}\right)-\left(\hat{s}_{A}, \hat{s}_{B}\right)}^{2}=\left\|\left(s_{A}-\hat{s}_{A}\right)+\alpha\left(s_{B}-\hat{s}_{B}\right)\right\|^{2} .
$$

Fig. 8 depicts the squared minimum distance as a function of channel parameter $\alpha$. It is obvious from this figure, that the minimum squared distance is highly resistant to the relative phase shift $(\angle \alpha)$ of the source-relay channels. Note that distance shortening at $|\alpha| \rightarrow 0$ is inevitable.

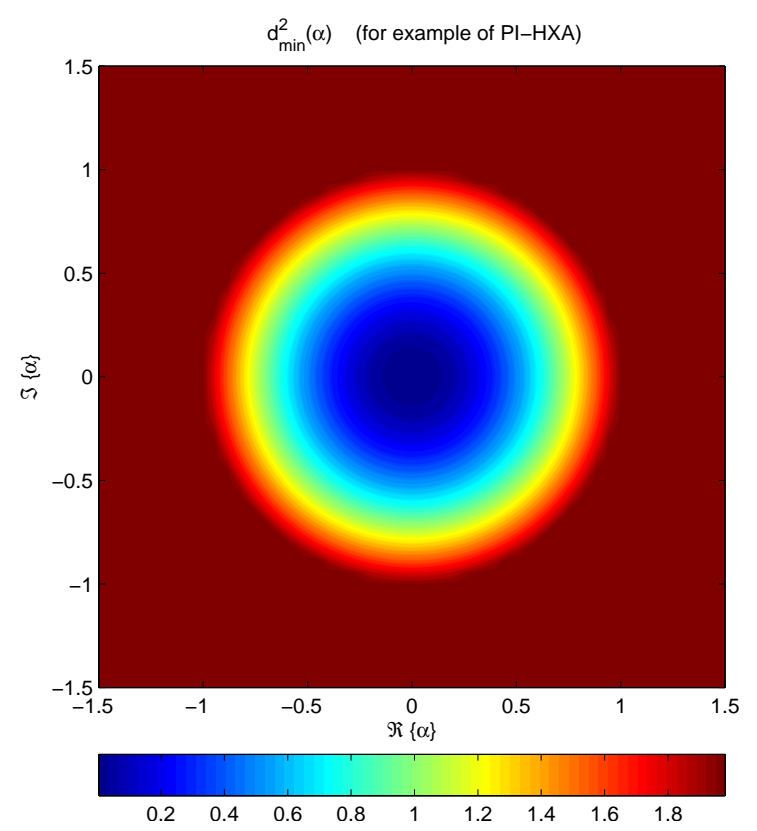

Fig. 8: Minimum squared distance as a function of the channel parameter (for the example of PI-HXA from Fig. 5) 


\section{Conclusion}

The importance of finding HXA resistant to channel parametrization (PI-HXA) was stated in [3]. It was observed there that some particular channel parametrization values have disastrous effects on the system performance (significant capacity degradation caused by a violation of the exclusive property). The first approach to PI-HXA design (E-PHXC design criteria [11]) has until now led only to orthogonal or non-zero mean HXA with unsatisfactory performance (capacity limited by classical MAC).

In this paper we have presented an example of a geometrical approach to PI-HXA design. Though the design was based on many simplifying assumptions, the numerical results show relatively high resistance of the capacity (Fig. 7) and the minimum distance (Fig. 8) to the channel parametrization. In addition, our setup requires no adaptation of the hierarchical exclusive mapping (unlike $[6,8]$ ). Hence the processing at the relay can always be kept transparent to both sources.

The numerical results presented in this paper show the viability of the layered approach to HXC design in 2-WRC with the HDF strategy, even for the case of parametric channels. Deriving systematic criteria for PI-HXA design is a topic for future work.

\section{Acknowledgement}

This work was supported by the FP7-ICT SAPHYRE project, by Grant Agency of the Czech Republic, project 102/09/1624, and by Ministry of Education, Youth and Sport of the Czech Republic, programme MSM6840770014, grant OC188 and by the Grant Agency of the Czech Technical University in Prague, grant No. SGS10/287/OHK3/3T/13.

\section{References}

[1] Yeung, R. W., Li, S.-Y. R., Cai, N., Zhang, Z.: Network Coding Theory. now Publishers, 2006.

[2] Sykora, J., Burr, A.: Hierarchical exclusive codebook design using exclusive alphabet and its capacity regions for $\mathrm{HDF}$ strategy in parametric wireless 2-WRC, in COST 2100 MCM, (Vienna, Austria), pp. 1-9, Sept. 2009. TD-09-933.

[3] Sykora, J., Burr, A.: Hierarchical alphabet and parametric channel constrained capacity regions for HDF strategy in parametric wireless 2-WRC, in Proc. IEEE Wireless Commun. Network. Conf. (WCNC), (Sydney, Australia), pp. 1-6, Apr. 2010.
[4] Baik, I.-J., Chung, S.-Y.: Network coding for twoway relay channels using lattices, in Proc. IEEE Internat. Conf. on Commun. (ICC), 2008.

[5] Popovski, P., Koike-Akino, T.: Coded bidirectional relaying in wireless networks, in Advances in Wireless Communications (V. Tarokh, ed.), Springer, 2009.

[6] Koike-Akino, T., Popovski, P., Tarokh, V.: Denoising maps and constellations for wireless network coding in two-way relaying systems, in Proc. IEEE Global Telecommun. Conf. (GlobeCom), 2008.

[7] Koike-Akino, T., Popovski, P., Tarokh, V.: Denoising strategy for convolutionally-coded bidirectional relaying, in Proc. IEEE Internat. Conf. on Commun. (ICC), 2009.

[8] Koike-Akino, T., Popovski, P., Tarokh, V.: Optimized constellations for two-way wireless relaying with physical network coding, IEEE J. Sel. Areas Commun., Vol. 27, p. 773-787, June 2009.

[9] Nam, W., Chung, S.-Y., Lee, Y. H.: Capacity bounds for two-way relay channels, in Proc. Int. Zurich Seminar on Communications, 2008.

[10] Erez, U., Zamir, R.: Achieving $1 / 2 \log (1+$ SNR $)$ on the AWGN channel with lattice encoding and decoding, IEEE Trans. Inf. Theory, Vol. 50, p. 2293-2314, Oct. 2004.

[11] Uricar, T., Sykora, J.: Extended design criteria for hierarchical exclusive code with pairwise parameter-invariant boundaries for wireless 2-way relay channel, in COST 2100 MCM, (Vienna, Austria), p. 1-8, Sept. 2009. TD-09-952.

[12] Uricar, T., Sykora, J.: Design criteria for hierarchical exclusive code with parameter invariant decision regions for wireless 2-way relay channel, Submitted for publication, 2010.

[13] Sykora, J.: Design criteria for parametric hierarchical exclusive constellation space code for wireless 2-way relay channel, in COST $2100 \mathrm{MCM}$, (Valencia, Spain), p. 1-6, May 2009. TD-09-855.

Tomáš Uřičář

E-mail: uricatom@fel.cvut.cz

Dept. of Radioelectronics

Faculty of Electrical Engineering

Czech Technical University in Prague

Technicka 2, 16627 Prague, Czech Republic 